\title{
Computational Power Conservation Technique Using Mobility Adaptation Method in MANET
}

\author{
Lawal Bello, Panos Bakalis, and Predrag Rapajic \\ Wireless and Mobile Communications Research Center \\ Faculty of Engineering and Science, University of Greenwich, Chatham Maritime, UK
}

\begin{abstract}
In this paper, a new technique is proposed for conserving power in mobile ad hoc wireless network. The technique is based on the existing on-demand ad hoc routing protocols with the addition of power model. The unique feature of the technique is that mobile nodes will be able to evaluate their power status to decide if they are fit for packet forwarding and reception. This is illustrated using computational approach supported by computer simulations over mobile ad hoc wireless network containing 18 mobile nodes. The proposed technique is incorporated into the Ad Hoc On demand Distance Vector (AODV) and Dynamic Source Routing (DSR) protocols. The results showed that using power saving technique reduces power consumption to more than $15 \%$ rate at which mobile nodes achieved with no much delay in the network as compared to the conventional network configured with AODV and DSR routing protocols.
\end{abstract}

\section{INTRODUCTION}

The spread of mobile computing and communication devices such as mobile phones, laptops, tablets is motivating a radical transformation in our information world. People are now migrating from the state of a personal computer age to a state where people can use their personal computer and at the same time connect with several other devices through which they can access all required information whenever the need arises. The nature of this interconnection between these devices makes wireless networks one of the suitable solutions for communication. As a result of this, the wireless network has been enormously supported in the past decade. Wireless networks can be categorized into different types, mobile ad hoc wireless network (MANET) is one of them. A mobile Ad hoc Wireless network is a group of mobile devices randomly connected together to communicate wirelessly without having any cabling network infrastructure, base stations or routers.

However, MANET is particularly vulnerable due to its fundamental characteristics such as open medium, constantly changing network topology, distributed and co-operative communication and inherently power constrained capabilities, which manifest in the exhaustible sources of power as reported in [1-3] which hold it from delivering the best desired performance. One of these challenges is the area of limited power. This issue is as a result of the fact that, the devices used in MANET are powered by batteries which are subjected to maximum life span before it drains out. The rate at which the power is used on the network is determined by the type of operations the network carries out. More so, the power consumption in MANET is directly proportional to the route length; this implies that if route length is increased, power consumption also increases. The major function each mobile node in MANET carries out involves transmission, reception, retransmission and beaconing; all these operations also determines how power is consumed. Power consumed by mobile nodes needs to be reduced in order to maintain network connectivity, avoid network portioning and also to provide power efficient operation. The applications these networks are used in areas where deployment of network cabling infrastructures are very difficult, owing to not having any centralized administration; such as disaster areas, battlefield, emergency and rescue places. This unique nature of MANET presents substantial challenges in developing optimum routing protocols $[4,5]$.

\section{MOTIVATION OF THE RESEARCH AS WELL AS TECHNICAL ISSUES}

The growth of wireless communication technologies has been exceptionally large in the last decade and it is predicted that the demand for these technologies will increase dramatically in the coming years.

Therefore, it is required to broaden the vision of wireless technologies by developing advance techniques at an appropriate layer of the wireless networking, particularly at the network layer of mobile ad hoc networks, where accurate and correct routing algorithm must be used. A little improvement in performance at the network layer has dramatically improved overall output of the 
wireless networks. To meet increasing demand, many efforts have been made in past research literature by using various power techniques to reduce the power consumption in MANET. The aim of the scheme is to reduce the routing power consumption rate, so that quality of service and performance throughput can be fully achieved during the communication. The computational complexity is also very important factor in designing algorithms for routing processing. complexity further increases in MANETs, particularly when network size grows and feasibility of implementation becomes very difficult. Most of the proposed power reduction schemes presented in the literature are too complex to implement in MANETs. Therefore, it it required to present a new scheme that is not only effective in all conditions of a communication channel but also computationally simple.

\section{RELATED WORK}

Mobile devices depend on batteries for power supply and since battery power is limited, power conservation depicts one of the utmost issues in designing algorithms for mobile devices [6]. Several works done in battery technology show that only slight improvement in the battery life are expected in the near future [7]. So, it is essential that power conservation should be optimized efficiently by identifying different mediums to reduce the usage of power without affecting the efficiency of the network.

Limitations on battery life and the extra power requirements for assisting network operations such as transmission or reception in each mobile node in MANET makes the issue of power conservation one of the main concerns in ad hoc networking. Power conservation techniques have been proposed at several levels of a mobile device including the physical layer transmissions, as well as operating system [8]. Takeuchi et al., in [9] observed from their work that power is wasted when wireless ad hoc interfaces are in idle state, and hence concluded that due to the fact that the interfaces remain in the ideal state for a long period of time, turning off the interfaces radio would reduce power consumption in the network. Experiment carried out by Zheng and Kravets in [10] has shown that the consumption of power in wireless ad hoc is marginally lower than that consumed in transmitting and receiving state. They also proposed an extensible on demand power management framework for ad hoc networks that adapts to traffic load. Each mobile node keeps soft state timers that observe routing control and data transmission to decide power management transitions. This process enables nodes to go off temporarily as supported by the MAC protocol. A prototype of the specified framework is then incorporated into the conventional IEEE 802.11 MAC protocol. Arulanandam and Parthasarathy in [11] proposed a power conservation scheme to reduce idle power consumption. The functionality of this scheme is not dependent on the design and operation of routing protocol. They compared power conservation patterns of both DSR and AODV under different condition. In order to derive the utilization of mobile nodes between the algorithms, the standard deviation of remaining power on the nodes after utilization was measured.

A lower standard deviation leads to a more balanced node utilization. From the comparisons made on the two algorithm based on their node utilization, AODV show slightly a more balanced utilization than the DSR. Arulanandam and Parthasarathy [11] concluded that DSR algorithm provides the most reliable combination of power conservation performance and data delivery performance. However, power conservation and control have become paramount issues that receive greater attentions in recent time [12].

The IEEE 802.11 power control mechanism is the basic platform most widely deployed when it comes to wireless networks power conservation protocols development $[4,5,12,13]$. But empirical information concerning the power consumption behaviour of well-known wireless network interfaces is not available $[5,13]$ and vendor device specifications do not provide enough information in a form that may be helpful to protocol developers. Therefore, power control design and evaluation of network protocols demand the knowledge of power consumption characteristics of actual wireless interfaces [5, 12]. Most researches in power conservation schemes has targeted wireless networks that are structured around base stations and centralized servers [13] which do not have the limitations associated with small, portable devices. By contrast, MANETs, as explained earlier do not operate with the services of such fixed network cabling infrastructure [13]. Therefore, it is cumbersome as well as challenging to design and develop power control strategies in an ad hoc fashion [14]. Some researchers have conducted practical experiments on the actual wireless devices such as Alsalih in [15], for instance, Laptop, PDA, etc. measuring the consumed power in a real time environment, which serves as experimental references for this design. Literatures [5,12] and [13] are some of those works. Power conservation in MANET, as a challenge is receiving the first and foremost responses because every other activity of the network depends on the ability of the network to 
maintain live connectivity, which is an exclusive role of the power source.

Therefore, the main contribution of this paper is to proposed a novel technique for MANETs that computes power less costly in terms of computation and conserves adaptively for proper transmission and reception of data packet.

\section{OVERVIEW OF ON DEMAND ROUTING PROTOCOLS}

\subsection{Dynamic Source Routing Protocol (DSR)}

Dynamic Source Routing is a simple, efficient and an On-demand routing protocol designed specifically for use in multi-hop wireless ad hoc networks that use source routing rather than the hop-byhop routing approach [16]. Each packet to be routed carries in its header a complete ordered list of nodes through which the packet passes. The advantage of this protocol is that intermediate nodes do not need to maintain up-to-date routing information in order to route the packets. Due to the on-demand characteristics of DSR, periodic route updates and neighbor detection are eliminated to minimize bandwidth consumption $[17,18]$. DSR has two basic mechanisms of operations, a Route Discovery process by flooding the network with route request (RREQ) packets to all its neighboring nodes containing the IP address of both sender and receiver in the packet header, whilst Route reply returns the route reply messages (RREP). The route reply contains the list of the best routes from the source initiator to the target destination.

\subsection{Ad Hoc on Demand Distance Vector Protocol (AODV)}

Ad Hoc On-Demand Distance Vector (AODV) is an on-demand routing protocol that combines the capabilities of both Dynamic Source Routing (DSR) and Destination-Sequenced Distance-Vector (DSDV). It uses the on-demand mechanisms of Route Discovery and Route Maintenance from DSR, in addition to the hop-by-hop routing sequence numbers and periodic beacons from DSDV as described by [19]. Mobile nodes requesting to forward packets to other mobile nodes will broadcast the route request (RREQ) packet to its neighbours which then forward the request to their neighbours until either the destination or an intermediate mobile node(s) with route(s) to the destination is located. AODV uses destination sequence numbers and broadcast IDs on each node to ensure all routes are loop-free and contain the most recent route information.

However, performance evaluation conducted on both AODV and DSR protocols in [16, 20-23], showed that AODV performs better than DSR and other proactive protocols in terms of throughput, end-to-end delay, and packets drop. The DSR performance is attributed to its characteristics of having multiple routes to other destinations. References [19,24-26] showed that, in a high complex network where mobility is very high, the DSR has high energy consumption in the entire network compared to its counterpart AODV, which losses energy due to broadcasting hello messages to update its routes. The proactive routing protocols use routing tables which are maintained via periodic updates from all other mobile nodes in the network, irrespective of the fact that, the network may not be active in terms of data traffic. Hence, AODV and DSR protocols were chosen as a genial candidate for carrying out this research.

\section{DESCRIPTION OF THE PROPOSED POWER CONSERVATION TECHNIQUES}

While considering the MANET's applications, it is important to modify the existing routing algorithm to specifically conserve power adaptively. The proposed model can be used on any of the on-demand routing algorithms. The setback to power management in ad hoc mobile communications is the choice of transmit power. Most of the research work was done in trying to establish the transmitted power of the mobile devices. For instance, the actual amount of power allotted to the transceiver of the mobile device is not actually included in vendor specification, and such a parameter is very important when it comes to protocol development and enhancement.

The typical transmission power of the CISCO Aironet 340 Access Point that utilizes power generating meter is in the range of $-10 \mathrm{dBm}(0.1 \mathrm{~mW})$ to $+30 \mathrm{dBm}(1 \mathrm{~W})$ is $32 \mathrm{~mW}(15 \mathrm{dBm})[27,28]$. This is the wireless LAN transmission power in laptops. However, that for typical cellular phone transmission power (Power Class 2 Mobiles) is $500 \mathrm{~mW}(27 \mathrm{dBm})$. Therefore, in this research work, the simulation set up is modelled with laptops as the mobile nodes, and the transmit power is implemented accordingly using the value of $32 \mathrm{~mW}$. The IEEE802.11 standard's power control management support power utilization in two modes:

1) Continuous Aware Mode and

2) Power Save Polling Mode. 
It is shown that IEEE $802.1 \mathrm{~b}$ in an idle network interface has a power consumption of $800 \mathrm{~mW}$. This is comparable to the power consumed while transmitting or receiving $(1000 \mathrm{~mW}-1300 \mathrm{~mW}$ respectively). However, in the sleeping mode case, the power consumption is $(66 \mathrm{~mW}-30 \mathrm{~mW})$. These values can vary depending on manufacturer and model. Researchers have explored this doze mode in order to extend the lifetime of networks in MANET. Among the literatures that used this mechanism as a basis for their researches are [4,5] and [14].

Therefore, the scheme designed is to enable the mobile nodes to avoid power drainage by maintaining a certain level of power in all possible routes. While no single mobile node is overused, the relationship among these metrics as a function is structured around how much power is incurred by any mobile node at a given time. Hence, the power consumed $P_{c}[\mathrm{~W}]$, as a function of idle power consumption, number of packets received and receive power is designed to give account of extent of usage of power at a given time in a network as shown in Eq. (1)

$$
\text { Power }_{\text {consumed }}=d P_{t x} / d t_{T i}+n \times P_{r x}
$$

where $d P_{t x}[\mathrm{~W}]$ is power consumption at idle mode, $\mathrm{n}$ is the total number of packets a node received at a given time. From [36], the received signal power $P_{r x}[\mathrm{~W}]$ can be written as

$$
P_{r x}=P_{t x} \times G_{t x} \times G_{r x} \times P_{d}
$$

where $G_{t x}, G_{r x}$ are the transmitter and receiver antenna gains and $P_{d}[\mathrm{~m}]$ is the propagation distance between mobile devices.

The proposed power model is designed in such a way that mobile nodes are able to evaluate their power status to decide if they are fit for packet forwarding and reception. These values are to be shared between the physical layer, Mac layer and Network layer (Cross layer interaction). The physical layer, Mac layer, sends the computed power values to the network routing layer, where it is stored and used for routing decision making process. The ability of a mobile node to be sustained, supported, upheld without being completely used up or destroyed; the sustainable power $S_{\text {plevel }}[\mathrm{w}]$ is computed as shown in Eq. (3).

$$
S_{\text {Plevel }}=P_{t x}-T_{p}
$$

where $T_{p}[\mathrm{w}]$ is the threshold power, given as $20 \%$ of $P_{t x}$.

Therefore, for successful transmission and reception of data packets the transmitting power in the mobile nodes must be greater than or equal to the sustainable power as shown in Eq. (4).

$$
P_{t x}>=S_{\text {plevel }}
$$

The procedure depicts the steps involved in the algorithm pseudo code representation, and gives a pictorial under-standing of the algorithm. At the beginning of the network operation, the communicating mobile node initiate and compute the power quantities needed to participate in the routing operations. The mobile node evaluates control statement. If the statement is true then, the mobile node will participate in the routing operation and send the packets to the desired destination else the mobile node re-compute the power values again. The process continues till end of simulation time. This is very important because the nodes will make their decisions concerning their participation in transmission at this stage and help to avoid power drainage by maintaining a certain level of power in all possible routes in the network.

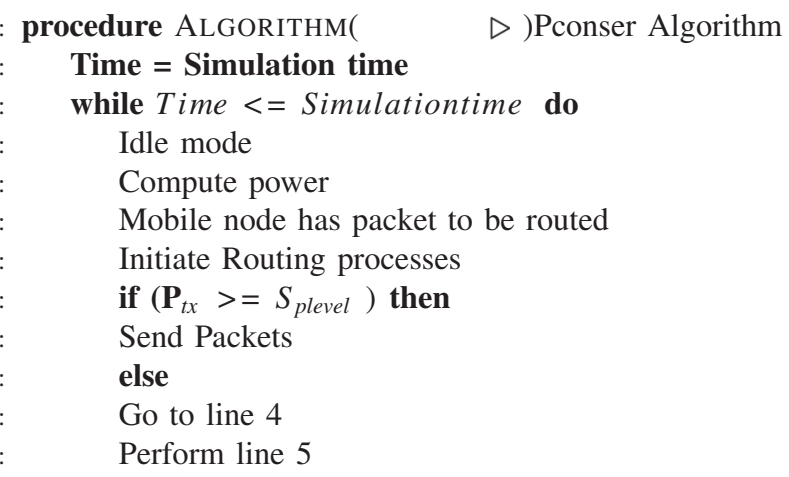

Figure 1: Power conservation algorithm. 
The $\mathrm{C}++$ code of the proposed power model was generated from the scheme which comprises of power metrics with their associated relationships, and the implementation was done in a cross-layer interaction between the MAC and the network layers. However, the power quantities needed by the mobile nodes for routing decisions were updated in the AODV and DSR packet header and the routing protocol will recognize the packets and evaluate the control statements of the inherent algorithms. The function block in the AODV and DSR route(s) will get the power values during the routing processes in the MANET transactions in order to evaluate the codes of the scheme designed.

\subsection{System and Propagation Model}

This section describes the system model and outlines basic assumptions used for the analysis. The radio environment is characterized by:

1) path loss,

2) shadowing and

3) multipath fading.

In this work, only path loss is considered as we assume a scenario in which an efficient antenna diversity-combining system is employed at the transmitting mobile nodes to eliminate the effects of multipath fading. Path loss occurs due to the decay of the intensity of a propagating radiowave and it requires an accurate estimation for proper determination of the electric field strength, signal-to-noise ratio, carrier-to-interference ratio, etc. [29]. This work considers only the free space path loss model, because other models, such as Okumura-Hata, COST 231-Hata, Walfish-Ikegami, COST 231-Walfish-Ikegami and Erceg are used for predicting path loss in mobile wireless systems designed to operate in the frequency band from $100 \mathrm{MHz}$ to $2000 \mathrm{MHz}$ [30-34]. Also assumed that no obstruction exists between a transmitter and a receiver, signals is transmitted through free space to the receiver located at a propagation distance $d[\mathrm{~m}]$. The free space path loss model used in this analysis is given by [35] as

$$
P_{d}=P_{t} G_{T x} G_{r x}\left(\frac{\lambda}{4 \pi d}\right)^{2}
$$

where $P_{d}[\mathrm{~W}]$ is the path loss in a free space, $P_{t}[\mathrm{~W}]$ is the transmitted signal power, $G_{t x}$ and $G_{r x}$ are the transmitter and receiver antenna gains and $\lambda$ is the carrier wavelength.

Since this scheme tries to enable the nodes to avoid power drainage by maintaining a certain level of power in all possible routes, the values which will be assigned to these quantities will reflect the objective of fair and controlled usage of power in order that nodes will continue to maintain the network for a longer period of time.

1) Transmit Power: The quantity of this metric has been established by research made in Section 5 which is $0.032 \mathrm{~W}$.

2) Idle Power Consumption: This quantity was assigned as a percentage of the transmit power, using the power usage at idle mode obtained in $[27,28]$. The value is taken to be $5 \%$ of the transmit power: Idle $e_{\text {power }}=5 \%$ of $P_{t x}$.

3) Threshold Power: The value of this parameter considers the residual power, after hectic network participation needed to ensure nodes connectivity till the end of a network scenario. The value is given as: $T_{p}=20 \%$ of $P_{t x}$.

4) Received Power: This value has a default formula set in the OPNET Modeller software. Although it is actually modifiable in the source codes, it will be deduced from the graphical result representation for the final computation.

- Number of Received Packets: This will also be determined from result.

\section{SIMULATION MODEL}

OPNET Modeller (version 17.1), developed by OPNET Technologies, is used for all simulations [37]. Details about the simulation model and environment are presented in the rest of this section.

\subsection{Simulation Parameters}

In order to validate the proposed power model, extensive simulations are conducted where the power expended by a network configured with either AODV or DSR protocol with and without the power model was compared. The simulation time is 500 seconds real time. Each simulation scenario was repeated 6 times, which enabled the simulation to converge for an accurate result. The basic parameters used for the simulations are summarized in Table 1. 
Table 1: Simulation parameters.

\begin{tabular}{lc}
\hline Parameters & Values \\
\hline Channel Type & Wireless Channel \\
Physical Characteristics & $802.11 \mathrm{n}$ \\
Data Rate & $6.5 \mathrm{Mbs}$ \\
Topology & $100 \mathrm{~m} \times 100 \mathrm{~m}$ \\
Routing Protocols & AODV, DSR \\
Number of Nodes & 18 \\
Transmit Power & 0.032 watt \\
Packet Size & $4096 \mathrm{bits}$ \\
Mobility Model & Random Way Point \\
Simulator & OPNET 17.1 version \\
Simulation Time & $500 \mathrm{sec}$ \\
Traffic Source & CBR \\
Speed & $0-20 \mathrm{~m} / \mathrm{sec}$ \\
Number of trial & Six $(6)$ \\
Packet Inter-arrival time & $0.25 \mathrm{~seconds}$ \\
\hline
\end{tabular}

\section{RESULTS AND DISCUSSION}

The goal of the evaluation is to show the effectiveness of the proposed power saving model. The performance evaluation was studied at every 100 real seconds of time not on an individual mobile node basis. The power model is incorporated into the two on demand routing protocols algorithms i.e., AODV and DSR protocols. In Figure 2, it is easy to see that the proposed power model reduces power consumption by more than $15 \%$ for the entire simulation time, compared to the network with the traditional network configured with either AODV or DSR protocol. The algorithm did not allow the mobile nodes to experience a surge in power dissipation as can be seen in Figure 2.

The algorithm maintained a steady control of power with crests of minimal power upsurge. However, in this model, the mobile nodes have been enhanced to refer to their residual power in order to make a decision concerning their participation in packet forwarding transactions. Therefore, they make sure that they have enough power prior to packet reception.

This quantity (power consumed) was computed, implemented and measured to verify the actual amount of power consumed by mobile nodes during the entire network duration. Comparison was made for the two scenarios to give a clearer knowledge of the performance difference. The power consumed was calculated using Eq. (1).

However, power was conserved at the expense of the throughput. The throughput was negatively affected by the proposed model as the throughput with the power model fell drastically as shown in Figure 3.

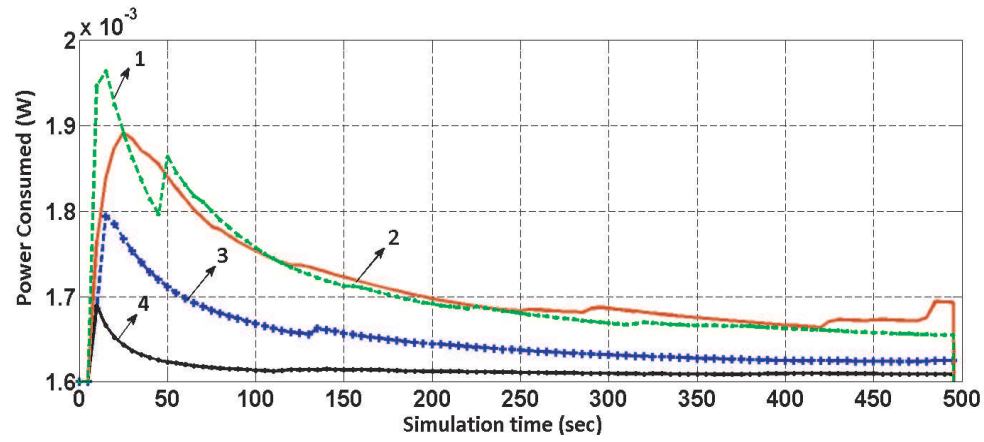

Figure 2: Power consumed comparison with and without the power model for the network configured with AODV and DSR protocols performance measure. 1) Power consumed AODV-Network configured with power model. 2) Power consumed AODV-Network configured without power model. 3) Power consumed DSR-Network configured without power model. 4) Power consumed DSR-Network configured with power model. 


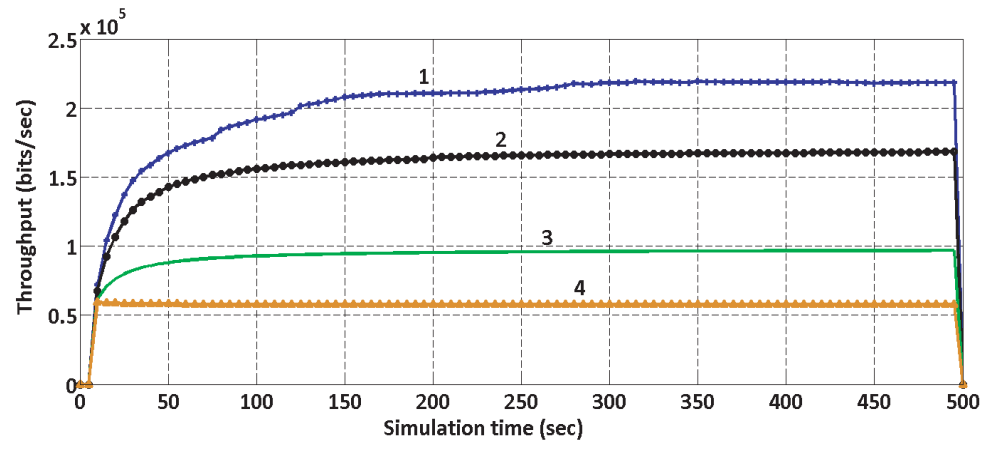

Figure 3: Throughput comparison with and without the power model for the network configured with AODV and DSR protocols performance measure. 1) Throughput AODV-Network configured without power model. 2) Throughput AODV-Network configured with power model. 3) Throughput DSR-Network configured with power model. 4) Throughput DSR-Network configured without power model.

As the algorithms repeat the routing search until the route(s) that satisfied the conditions, then the packet is routed to the desired destination(s) which can lead to the time to live (TTL) of the routing protocols expiration. For that, packet will be dropped and consequently effect the throughput performance of the network as shown in Figure 3.

At the beginning of the simulation, the scenario without power model delivered more than 200000 bits and 150000 of bits per second with the power model, all configured with AODV protocol, compared to the other network scenario without power model delivered less than 100000 bits of data per second and more than 50000 bits with the scheme incorporated all configured with DSR protocol. The initial rise of throughput for all the scenarios is due to a route discovery process initiated by the routing protocols. The significant differences between the modified and unmodified network is that, the delivery of packets to the desired destination depends on the stated power model condition. However, the traditional network configured with AODV and DSR protocols will continue to operate conventionally whether the condition is met or not and so packets will be dropped due to the inefficiency of the power resource of the mobile node to convey the packets to the desired destination. Therefore, the network configured with AODV protocol outperformed the network configured with DSR protocol and this is due to the fact that only the stable route(s) are used by the AODV forwarding algorithm for routing the packets.

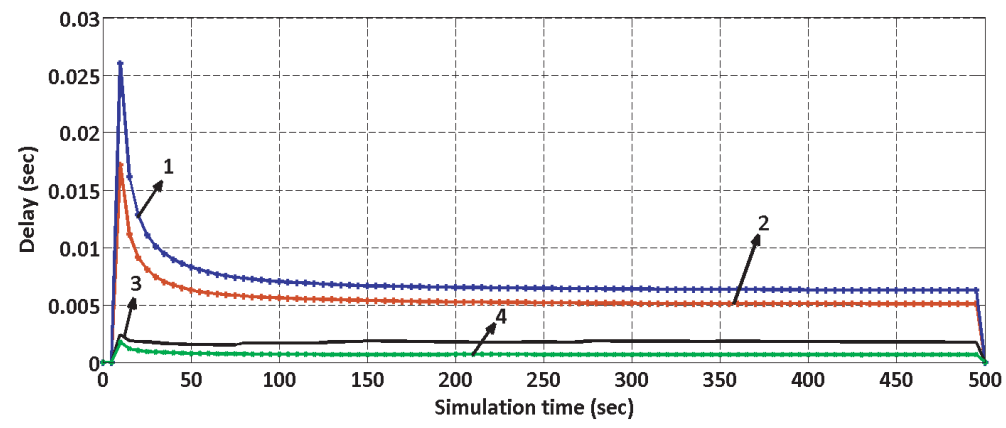

Figure 4: Delay comparison with and without the power model for the network configured with AODV and DSR protocols performance measure. 1) Delay DSR-Network configured without power model. 2) Delay DSR-Network configured with power model. 3) Delay AODV-Network configured without power model. 4) Delay AODV-Network configured with power model.

Figure 4 shows the delay curves for the network with and without the power model. At the start of the simulation, the network configured with AODV protocol experienced a huge delay of an average of about 0.026 seconds without the power model and 0.017 seconds with power model. Meanwhile, the other network scenario configured with DSR protocol experienced a little delay of about of 0.0035 seconds without power model and 0.002 seconds with the power model.

However, the initial rise in delay is due to the initial stage of route discovery processes of the routing protocols. But the proposed power model does not have much of an effect on the 
performance scenarios with power model as shown in Figure 4. Hence, less delay was observed in the network scenario configured with DSR protocol with least delay recorded with the power model. From the delay curves, the network using AODV protocol with the power model recorded less delay compared to the network with AODV without the power model. All these margin differences are attributed to the differences in routing operations of the routing protocols.

\section{CONCLUSION}

In this paper, we proposed a novel power conservation algorithm for MANETs that computes and conserves power by evaluating the mobile nodes power status to decide if they are fit for packet reception and forwarding. We computed,implemented and measured the performance of this scheme and showed that more than $15 \%$ of the power was conserved. The unique aspect of our proposed scheme is that it achieves these power savings without experiencing huge delay as compared to the coventional network configured with AODV and DSR routing protocols. To derive the utilization of the mobile nodes in the network, standard deviation of the network performance metric after utilization was measured. The comparison, shows that a lower standard deviation leads to a more balanced mobile node utilization in the network.

Therefore, we conclude that using the proposed power model algorithm to establish the routing processes, saved mobile nodes from dying as a result of power depletion during the network routing operation. Our protocol performs better in terms of power saving, delay reduction as compared to work in [38-40], despite the fact that they have used different simulation parameters as well as routing protocols. However, Chen in [40] agreed that their techniques was extremely expensive in terms of power consumption and this warrants investigation into more technique that can save more power of the mobile nodes in the network.

\section{ACKNOWLEDGMENT}

The authors would like to thank the Wireless and Mobile Communications Research Centre for supporting the research, the School of Engineering and Science, University of Greenwich, United Kingdom and OPNET Technology Group now (Riverbed Technology) for providing the software used for the simulations.

\section{REFERENCES}

1. Gallina, L., S. Hamadou, A. Marin, and S. Rossi, "A framework for throughput and energy efficiency in mobile ad hoc networks," Oct. 2011.

2. Mahimkar, A. and R. Shyamasundar, "S-mecra: A secure energy-efficient routing protocol for wireless ad hoc networks," Vol. 4, Oct. 2004.

3. Nikolakopoulos, G., A. Panousopoulou, and A. Tzes, "Manet-issues and their effects on control applications," Vol. 2, Sep. 2005.

4. Cano, J. and P. Manzoni, "A low power protocol to broadcast real-time data traffic in a clustered ad hoc network," Vol. 5, 2011.

5. Hsu, H.-Y. and A. R. Hurson, "Pean: A probabilistic energy aware neighbor monitoring protocol for mobile ad hoc networks," May 2007.

6. Forman, G. and J. Zahorjan, "The challenges of mobile computing," Proceedings of the IEEE Computer Society, 38-47, Apr. 1994.

7. Sheng, S., A. Chandrakasan, and R. Brodersen, "A portable multimedia terminal," Proceedings of the IEEE Communications Magazine, 64-75, Dept. of Electr. Eng. and Comput. Sci. and California Univ. and Berkeley, Dec. 1992.

8. Jones, C., K. Sivalingam, P. Agarwal, and J. Chen, "A survey of energy efficient network protocols for wireless and mobile networks," ACM: Wireless Networks, Vol. 7, Jan. 2011.

9. Takeuchi, S., Yamazaki, K. Sezaki, and Y. Yasuda, "An improved power saving mechanism for MAC protocol in ad hoc networks," Proceedings of the Global Telecommunications Conference, 2004. GLOBECOM'04. IEEE, 2791-2796, Grad. Sch. of Sci. Eng., Waseda Univ., Tokyo, Japan, Dec. 2004.

10. Zheng, R. and R. Kravets, "On-demand power management for ad hoc networks," INFOCOM 2003. Twenty-Second Annual Joint Conference of the IEEE Computer and Communications. IEEE Societies, 481-491, Dept. of Comput. Sci., Illinois Univ., Urbana, IL, USA, Apr. 2003.

11. Arulanandam, K. and B. Parthasarathy, "A new energy level efficiency issues in manet," International Journal of Reviews in Computing, 2009. 
12. He, Y., R. Yuan, X. Ma, and J. Li, "The IEEE 802.11 power saving mechanism: An experimental study," Apr. 2008.

13. Feeney, L. and M. Nilsson, "Investigating the energy consumption of a wireless network interface in an ad hoc networking environment," Vol. 3, 2001.

14. Liu, W., Y. Zhang, and Y. Fang, "Delar: A device-energy-load aware relaying framework for heterogeneous mobile ad hoc networks," IEEE Journal on Selected Areas in Communications, Vol. 29, No. 8, Sep. 2011.

15. Alsalih, W., S. Akl, and H. Hassanein, "Energy-aware task scheduling: Towards enabling mobile computing over manets," Apr. 2005.

16. Raju, S. R. and J. Mungara, "Performance evaluation of ZRP over AODV and DSR in mobile adhoc networks using qualnet," European Journal of Scientific Research, Vol. 45, 658-674, 2010.

17. Johnson, B. D., A. D. Maltz, and J. Broch, "The dynamic source routing protocol for multihopwireless ad hoc networks," Networks. Computer Science Department Carnegie Mellon, PA 15213-3891, University, Pittsburgh.

18. Basagni, S., M. Conti, S. Giordano, and I. Stojmenovic, Mobile Ad Hoc Networking, IEEE Press, 2004.

19. Yang, S. Z., B. Qi, and J. He, "Simulation comparison of four wireless ad hoc routing protocols," Information Technology Journal, Vol. 3, No. 3, 219-226, 2004.

20. Qasim, N., F. Said, and H. Aghvami, "Mobile ad hoc networking protocols evaluation through simulation for quality of service," IAENG International Journal of Computer Science, Vol. 36, No. 1, 2009.

21. Kumar, B. R. A., L. C. Reddy, and P. S. Hiremath, "Performance comparison of wireless mobile ad hoc network routing protocols," IJCSNS International Journal of Computer Science and Network Security, Vol. 8, No. 1, 2008.

22. Broch, J., A. D. Maltz, B. D. Johnson, Y.-C. Hu, and J. Jetcheva, "A performance comparison of multi-hop wireless ad hoc network routing protocols," Networks. Computer Science Department Carnegie Mellon, PA 15213-3891, University, Pittsburgh, 1998.

23. Rahman, M. A., M. S. Islam, and A. Talevski, "Performance measurement of various routing protocols in ad hoc network," Proceedings of the International MultiConference of Engineers and Computer Scientist, Vol. I, Hong Kong, 2009.

24. Boukerche, A., Algorithm and Protocls for Wireless and Mobile Ad Hoc Networks, Wiley Seris on Parallel and Distributed Computing.

25. Quakil, L., S. Senouci, and G. Pujolle, "Performance comparison of ad hoc routing protocols based on energy consumption," Ambience Workshop, Torino, Italy, 2002.

26. Zhao, Q., L. Tong, and D. Cousil, "Energy-aware adaptive routing for large-scale ad hoc networks: Protocols and performance analysis," IEEE Transactions on Mobile Computing, Vol. 6, No. 9, 1048-1059, Sep. 2007.

27. I. Cisco Systems, "Wireless, LAN (WLAN): Rf power values," 2008.

28. Berger, H. and A. Tsaliovich, "Unlicensed pcs product emc compliance measurement rationale and alternatives," Aug. 1996.

29. Nešković, A., N. Nešković, and D. Paunocć, "Macrocell electric field strength prediciton model based upon artificial neural networks," Vol. 20, No. 6, 1170-1176, Aug. 2002.

30. Okumura, Y., E. Ohmori, T. Kawano, and K. Fukua, "Field strength and its variability in uhf and vhf land-mobile radio service," Rev. Elec. Commun. Lab., Vol. 16, No. 9, 1968.

31. Hata, M., "Empirical formula for propagation loss in land mobile radio services," Vol. VT-29, No. 3, 317-325, Sep. 1980.

32. Walfisch, J. and H. L. Bertoni, "A theoretical model of uhf propagation in urban environments," Vol. 36, No. 12, 1788-1796, Dec. 1988.

33. Erceg, V., L. J. Greenstein, S. Y. Tjandra, S. R. Parkoff, A. Gupta, B. Kulic, and A. A. Julius, "An emprically based path loss model for wireless channels in suburban environment," Vol. 17, No. 7, 1205-1211, Jul. 1999.

34. Nisirat, M. A., M. Ismail, L. A. Nissirat, and S. AlKhawaldeh, "A terrain roughness correction factor for hata path loss model at $900 \mathrm{MHz}$," Progress In Electromagnetics Research C, Vol. 22, 11-22, 2011.

35. Goldsmith, A., Wireless Communication, 31-35, Cambridge University, New York, NY, 2005. 
36. Zanca, G., F. Zorzi, A. Zanella, and M. Zorzi, "Experimental comparison of rssi-based localization algorithms for indoor wireless sensor networks," Workshop on Real-World Wireless Sensor Networks (RealWSN'08), Glasgow, UK, Apr. 2008.

37. "Opnet simulator," http://www.opnet.com/.

38. Singh, S., M. Woo, and C. S. Raghavendra, "Power-aware routing in mobile ad hoc networks," Proceedings of the 4th Annual ACM/IEEE International Conference on Mobile Computing and Networking Dallas, 181-190, 1998.

39. Ramachandran B. and S. Shanmugavel, "Received signal strength-based cross-layer designs for mobile ad hoc networks," IETE Technical Review, Vol. 25, No. 4, 2008.

40. Chen, B., K. Jamieson, and H. Balakrishnan, "Span: An energy-efficient coordination algorithm for topology maintenance in ad hoc wireless networks," Proceedings of the 7th ACM MOBICOM, Rome, Italy, Jul. 2001. 